\title{
Risk factors and survival of patients with permanent pacemaker implantation after heart transplantation
}

\author{
Rasmus Rivinius ${ }^{1,2,3}$, Matthias Helmschrott ${ }^{1}$, Ann-Kathrin Rahm ${ }^{1,2,3}$, Fabrice F. Darche ${ }^{1,2}$, \\ Dierk Thomas $^{1,2,3}$, Tom Bruckner ${ }^{4}$, Andreas O. Doesch ${ }^{1,5}$, Philipp Ehlermann ${ }^{1,3}$, Hugo A. Katus ${ }^{1,2,3}$, \\ Edgar Zitron ${ }^{1,2,3}$
}

${ }^{1}$ Department of Cardiology, Angiology and Pneumology, ${ }^{2}$ Heidelberg Center for Heart Rhythm Disorders (HCR), Heidelberg University Hospital, Heidelberg, Germany; ${ }^{3}$ German Center for Cardiovascular Research (DZHK), Partner Site Heidelberg/Mannheim, Heidelberg, Germany; ${ }^{4}$ Institute for Medical Biometry and Informatics, University of Heidelberg, Heidelberg, Germany; ${ }^{5}$ Department of Pneumology and Oncology, Asklepios Hospital, Bad Salzungen, Germany

Contributions: (I) Conception and design: R Rivinius, M Helmschrott, AK Rahm, E Zitron; (II) Administrative support: R Rivinius, D Thomas, P Ehlermann, HA Katus, E Zitron; (III) Provision of study materials or patients: R Rivinius, M Helmschrott, AK Rahm; (IV) Collection and assembly of data: R Rivinius, M Helmschrott, AK Rahm, FF Darche, T Bruckner; (V) Data analysis and interpretation: All authors; (VI) Manuscript writing: All authors; (VII) Final approval of manuscript: All authors.

Correspondence to: Dr. Rasmus Rivinius. Department of Cardiology, Angiology and Pneumology, Heidelberg University Hospital, Im Neuenheimer Feld 410, 69120 Heidelberg, Germany. Email: rasmus.rivinius@med.uni-heidelberg.de.

Background: Permanent pacemaker (PPM) implantation after heart transplantation (HTX) may be required due to severe bradycardia. The aim of this study was to investigate the risk factors, indications, perioperative outcomes and complications of PPM implantation after HTX as well as the underlying effect on post-transplant mortality including causes of death.

Methods: This registry study included 621 patients receiving HTX at Heidelberg Heart Center between 1989 and 2018. Patients were stratified by PPM implantation after HTX. Data analysis of risk factors for PPM implantation included donor and recipient demographics, post-transplant medication, mortality, and causes of death.

Results: Thirty-six patients (5.8\%) received PPM implantation after HTX, 12 (33.3\%) with early PPM and 24 (66.7\%) with late PPM. Indications for PPM implantation after HTX included sinus node dysfunction (SND) $(n=15 ; 41.7 \%)$ and atrioventricular block (AVB) (n=21; 58.3\%). Multivariate analysis revealed recipient body mass index (BMI) [hazard ratio (HR): 1.10; confidence interval (CI): 1.01-1.21; $\mathrm{P}=0.03$ ], donor age (HR: 1.07; CI: 1.03-1.10; $\mathrm{P}<0.01$ ), and biatrial HTX (HR: 2.63; CI: 1.22-5.68; P=0.01) as significant risk factors for PPM implantation after HTX. Kaplan-Meier estimator displayed a statistically significant inferior 5-year post-transplant survival among patients with early PPM after HTX in comparison to patients with late PPM or no PPM after HTX $(\mathrm{P}<0.01)$ along with a higher percentage of death due to infection $(\mathrm{P}<0.01)$.

Conclusions: Multivariate risk factors for PPM implantation after HTX include recipient BMI, donor age, and biatrial HTX. Early PPM implantation after HTX is associated with increased 5-year post-transplant mortality due to infection.

Keywords: Biatrial; sinus node dysfunction (SND); bradycardia; mortality; pacing

Submitted Jul 29, 2019. Accepted for publication Oct 08, 2019.

doi: $10.21037 /$ jtd.2019.11.45

View this article at: http://dx.doi.org/10.21037/jtd.2019.11.45

(C) Journal of Thoracic Disease. All rights reserved. 


\section{Introduction}

Cardiac rhythm disorders after heart transplantation (HTX) are frequent and may manifest in different ways either as tachyarrhythmias or as bradyarrhythmias (1-3). Common post-transplant bradyarrhythmias comprise sinus node dysfunction (SND) and atrioventricular block (AVB) which may require permanent pacemaker (PPM) $(4,5)$. Bradycardic rhythm disorders after HTX are often transient and improve over time, with reported rates of PPM implantation after HTX varying between $3.5 \%$ and $20.5 \%$ $(6,7)$. A large analysis of the United Network for Organ Sharing (UNOS) database showed that more than $10 \%$ of all patients received a PPM after HTX (5).

The initial standard technique for HTX, also known as the biatrial technique, has been associated with elevated rates of PPM implantation due to a continuous circular suture of the right donor and recipient atrium with possible sinus node injury $(2,4,8-10)$. In contrast, the bicaval technique uses two donor-to-recipient vena cava anastomoses preserving the right atrial (RA) integrity and the sinus node $(2,4)$.

Regarding the analysis of further risk factors, complications, and long-term outcomes of PPM implantation after HTX, there is only a minority of studies with a population of more than 500 patients and only one multi-center study investigating the need for PPM implantation after HTX (3-5,11-15). Additionally, studies yielded inconsistent results due to considerably differences in design, analyzed parameters, and length of followup. Moreover, the vast majority of the existing literature is outdated, as most studies were published in the 1990s (3,16-39) and in the 2000s (4,6,7,40-43).

Thus, given the need for new studies in this area of research, the aim of this large registry study was to investigate the risk factors, indications, perioperative outcomes and complications of PPM implantation after HTX as well as the underlying effect on post-transplant mortality including causes of death.

\section{Methods}

\section{Patients}

The performance of this study was in accordance with the ethical principles for medical research of the Declaration of Helsinki. Approval was given by the ethics committee of the University of Heidelberg (ethical approval number: S-286/2015, date of ethical approval: 22-06-2015). This study included all adult patients ( $\geq 18$ years) receiving HTX at Heidelberg Heart Center between 1989 and 2018 except for patients with repeated HTX. Four patients with implantable cardioverter defibrillator (ICD) implantation after HTX due to ventricular tachycardia and no requirement for pacing were also excluded. Written informed consent was obtained from patients for inclusion in the Heidelberg HTX Registry allowing the clinical and scientific use of data. According to the ethical approval, no additional written informed consent was required for this registry study as only routine clinical data were analyzed (2,44-49).

All available medical records were screened for PPM implantation after HTX and patients were accordingly stratified into the following two groups: Patients with PPM implantation after HTX ("PPM after HTX") and patients without PPM implantation after HTX ("No PPM after HTX"). Then, patients with PPM implantation after HTX were further subdivided into patients with PPM implantation $\leq 1$ year after HTX (early PPM after HTX) and patients with PPM implantation $>1$ year after HTX (late PPM after HTX).

\section{Follow-up}

Follow-up after HTX was performed according to the usual standard of care at Heidelberg Heart Center. As part of HTX surgery, patients received a temporary pacemaker system consisting of an external pacing box and two epicardial pacing leads which were placed on the right atrium and ventricle. The epicardial pacing leads routinely remained in situ for around 10 days after HTX. During the initial hospital stay, 12-lead electrocardiography (ECG) was regularly performed and in case of any suspected arrhythmic disorder. Before discharge, patients routinely had a 24-hour-holter-recording (2,44-49).

After the initial hospital stay, patients were followedup monthly during the first 6 months after HTX, then bimonthly between month 6 to 12 after HTX, and thereafter routinely three to four times annually. Routine followup included medical history, physical examination, ECG, echocardiography, endomyocardial biopsy, and blood tests including immunosuppressive drug monitoring $(2,44-51)$.

\section{Post-transplant medication}

Patients after HTX initially received an anti-thymocyte globulin-based immunosuppression induction therapy. The 
initial standard immunosuppressive drug regimen at the beginning of the study period consisting of cyclosporine A (CsA) and azathioprine (AZA) was subsequently switched to CsA and mycophenolate mofetil (MMF) from 2001 onward. Since 2006, tacrolimus (TAC) and MMF were routinely used as initial immunosuppressive drug therapy. Steroids (prednisolone) were tapered incrementally during the first post-transplant months and discontinued finally 6 months after HTX if possible (2,44-49).

\section{Statistical analysis}

SAS statistical software (Version 9.4, SAS Institute, Cary, NC, USA) was used for analysis of data. Data were given as mean \pm standard deviation (SD) or as count (n) and percentage (\%). Measures of association [mean difference (MD) or hazard ratio (HR)] with $95 \%$ confidence interval (CI) were applied for results. Student's $t$-test was used for continuous variables and chi-squared test was applied for categorical variables. Kaplan-Meier estimator was employed to graphically display 5-year survival after HTX. Extensive univariate analyses were performed to test for differences between groups covering recipient data, previous openheart surgery, principal diagnosis for HTX, donor data, perioperative data, and medication after HTX including immunosuppressive drug therapy (2,44-49).

In addition, a multivariate analysis (Cox regression model) was conducted to analyze the influence of the following five clinically relevant parameters which were statistically significant in the univariate analysis between patients with and without PPM implantation after HTX: recipient age, recipient body mass index (BMI), recipient arterial hypertension, donor age, and biatrial HTX. In order to avoid biased regression coefficients and to ensure a stable number of events (patients with PPM implantation after HTX) per analyzed variable, we did not include further parameters in this multivariate analysis (2,44-49).

In patients with PPM implantation after HTX, data regarding device surgery and outcomes of PPM implantation after HTX were further investigated including type and position of PPM, perioperative data, perioperative complications, and causes of death within 5 years after HTX. Furthermore, a sensitivity analysis was carried out to test the robustness of the study results using a subgroup of patients with TAC and MMF as immunosuppressive drug regimen as the immunosuppressive drug therapy was switched from $2006(2,44-49)$.

\section{Results}

\section{Baseline characteristics}

In this registry study including 621 patients, 36 patients (5.8\%) required PPM implantation after HTX. Hereof, 12 patients $(33.3 \%)$ had a PPM $\leq 1$ year after HTX (early PPM after HTX) and 24 patients $(66.7 \%)$ received a PPM >1 year after HTX (late PPM after HTX).

Patients with PPM implantation after HTX had a higher recipient age $(55.0 \pm 6.5$ versus $51.8 \pm 10.5$ years; $M D$ : 3.2 years, $95 \%$ CI: $0.8-5.6$ years, $\mathrm{P}=0.01)$, a higher recipient BMI $\left(26.4 \pm 4.1\right.$ versus $24.8 \pm 3.9 \mathrm{~kg} / \mathrm{m}^{2} ; \mathrm{MD}: 1.6 \mathrm{~kg} / \mathrm{m}^{2}$, $95 \%$ CI: $\left.0.2-3.0 \mathrm{~kg} / \mathrm{m}^{2}, \mathrm{P}=0.03\right)$, and a higher rate of recipient arterial hypertension $(72.2 \%$ versus $53.5 \%$; $\mathrm{MD}$ : 18.7\%; 95\% CI: $3.5-33.9 \%, \mathrm{P}=0.03)$ than patients without PPM after HTX. In terms of donor data, patients with PPM implantation after HTX showed a higher donor age $(46.7 \pm 11.8$ versus $40.7 \pm 13.5$ years; $\mathrm{MD}$ : 6.0 years, $95 \% \mathrm{CI}$ : $1.8-10.2$ years, $\mathrm{P}<0.01$ ), whereas there was no significant difference regarding male donor sex or donor BMI. No further donor data were available for this study.

Analysis of perioperative data revealed a higher percentage of biatrial HTX in patients with PPM implantation after HTX (41.7\% versus $25.1 \%$; MD: $16.6 \%, 95 \%$ CI: $0.2-33.0 \%, \mathrm{P}=0.03)$ and accordingly a higher degree of bicaval HTX in patients without PPM implantation after HTX (74.9\% versus 58.3\%; MD: 16.6\%, $95 \%$ CI: $0.2-33.0 \%, P=0.03)$. No statistically significant differences between groups could be detected in the remaining parameters. Baseline characteristics are presented in Table 1.

\section{Initial medication after $H T X$}

Comparison of immunosuppressive medication displayed no statistically significant differences between both groups regarding the use of CsA, TAC, Aza, or MMF. Additionally, there were no statistically significant differences in the administration of acetylsalicylic acid, beta-blockers, ivabradine, calcium channel blockers, angiotensin-converting-enzyme (ACE) inhibitors/sartans, or statins. An overview of the initial medication after HTX is given in Table 2.

\section{Multivariate analysis of risk factors for PPM implantation after HTX}

Multivariate analysis of risk factors for PPM implantation 
Table 1 Baseline characteristics

\begin{tabular}{|c|c|c|c|c|c|}
\hline Parameter & PPM $(n=36)$ & No PPM (n=585) & Difference & $95 \% \mathrm{Cl}$ & $P$ value \\
\hline Age (years), mean $\pm S D$ & $55.0 \pm 6.5$ & $51.8 \pm 10.5$ & 3.2 & $0.8-5.6$ & $0.01^{*}$ \\
\hline Male sex, n (\%) & $27(75.0)$ & $460(78.6)$ & 3.6 & $-10.9-18.1$ & 0.61 \\
\hline Body mass index $\left(\mathrm{kg} / \mathrm{m}^{2}\right)$, mean $\pm \mathrm{SD}$ & $26.4 \pm 4.1$ & $24.8 \pm 3.9$ & 1.6 & $0.2-3.0$ & $0.03^{*}$ \\
\hline Arterial hypertension, n (\%) & $26(72.2)$ & $313(53.5)$ & 18.7 & $3.5-33.9$ & $0.03^{*}$ \\
\hline Dyslipidemia, n (\%) & $26(72.2)$ & $368(62.9)$ & 9.3 & $-5.8-24.4$ & 0.26 \\
\hline Diabetes mellitus, $\mathrm{n}(\%)$ & $14(38.9)$ & $196(33.5)$ & 5.4 & $-11.0-21.8$ & 0.51 \\
\hline Renal insufficiency^, n (\%) & $21(58.3)$ & 335 (57.3) & 1.0 & $-15.5-17.5$ & 0.90 \\
\hline Overall open heart surgery, n (\%) & $10(27.8)$ & $171(29.2)$ & 1.4 & $-13.7-16.5$ & 0.85 \\
\hline CABG surgery, n (\%) & $5(13.9)$ & $72(12.3)$ & 1.6 & $-10.0-13.2$ & 0.78 \\
\hline Congenital, valvular or ventricular surgery, $\mathrm{n}(\%)$ & $5(13.9)$ & $65(11.1)$ & 2.8 & $-8.8-14.4$ & 0.61 \\
\hline VAD surgery, $\mathrm{n}(\%)$ & $3(8.3)$ & $44(7.5)$ & 0.8 & $-8.5-10.1$ & 0.86 \\
\hline \multicolumn{6}{|l|}{ Principal diagnosis for HTX } \\
\hline Ischemic CMP, n (\%) & $12(33.3)$ & $194(33.2)$ & 0.1 & $-15.8-16.0$ & 0.98 \\
\hline Non-ischemic CMP, n (\%) & $21(58.3)$ & $309(52.8)$ & 5.5 & $-11.1-22.1$ & 0.52 \\
\hline Valvular heart disease, $\mathrm{n}(\%)$ & $2(5.6)$ & $32(5.5)$ & 0.1 & $-7.6-7.8$ & 0.98 \\
\hline \multicolumn{6}{|l|}{ Perioperative data } \\
\hline Transplant sex mismatch, n (\%) & $16(44.4)$ & $263(45.0)$ & 0.6 & $-16.2-17.4$ & 0.95 \\
\hline Ischemic time (min), mean $\pm \mathrm{SD}$ & $217.8 \pm 72.9$ & $222.8 \pm 68.3$ & 5.0 & $-20.6-30.6$ & 0.70 \\
\hline Ischemic time $\geq 240 \mathrm{~min}, \mathrm{n}(\%)$ & $15(41.7)$ & $241(41.2)$ & 0.5 & $-16.1-17.1$ & 0.96 \\
\hline Biatrial HTX, n (\%) & $15(41.7)$ & $147(25.1)$ & 16.6 & $0.2-33.0$ & $0.03^{*}$ \\
\hline Bicaval HTX, n (\%) & $21(58.3)$ & $438(74.9)$ & 16.6 & $0.2-33.0$ & $0.03^{*}$ \\
\hline
\end{tabular}

^, GFR $<60 \mathrm{~mL} / \mathrm{min} / 1.73 \mathrm{~m}^{2}$; ${ }^{*}$, statistically significant $(\mathrm{P}<0.05)$. CABG, coronary artery bypass graft; CMP, cardiomyopathy; Cl, confidence interval; GFR, glomerular filtration rate; HTX, heart transplantation; n, number; PPM, permanent pacemaker; SD, standard deviation; VAD, ventricular assist device. 
Table 2 Initial medication after HTX

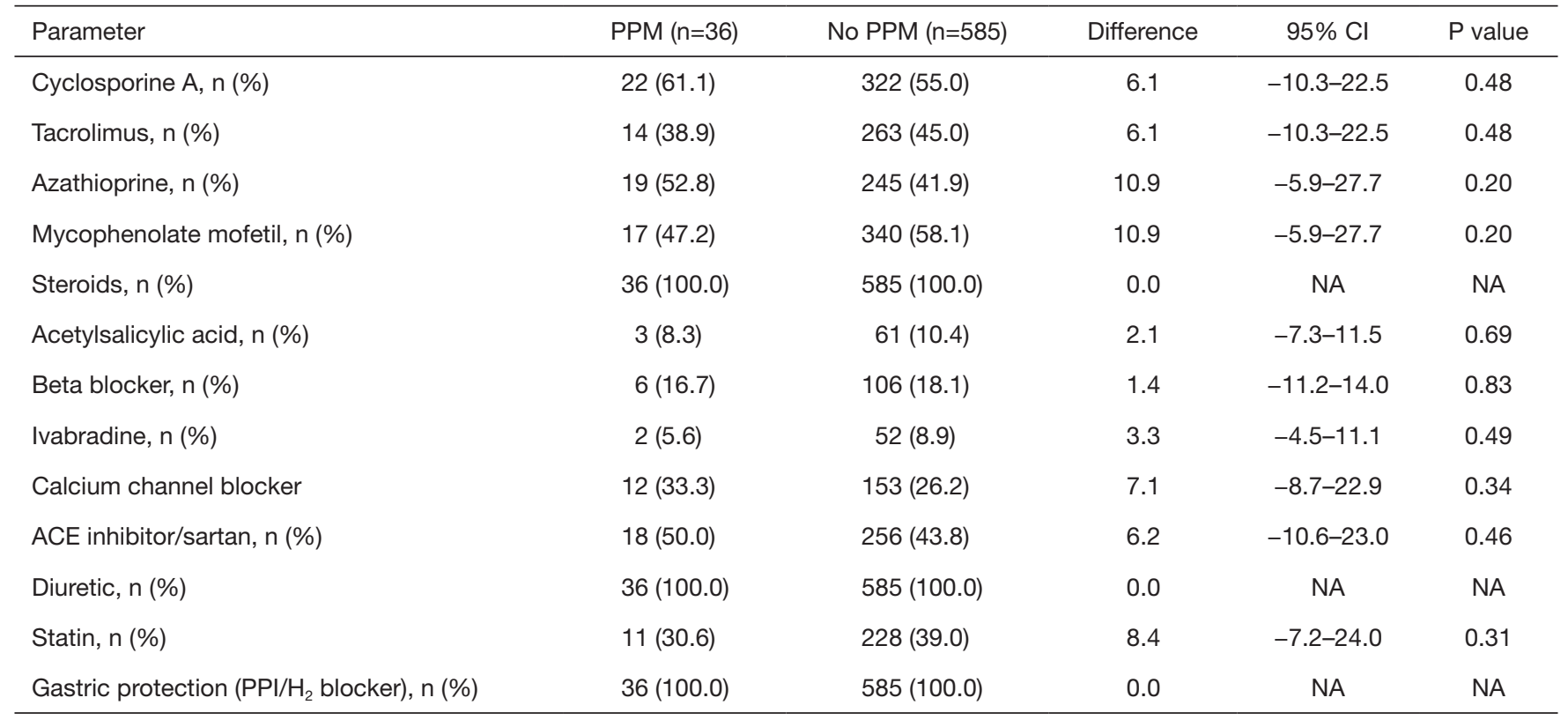

ACE inhibitor, angiotensin-converting-enzyme inhibitor; $\mathrm{Cl}$, confidence Interval; $\mathrm{HTX}$, heart transplantation; $\mathrm{H}_{2}$ blocker, histamine receptor blocker; n, number; NA, not applicable; PPI, proton pump inhibitor; PPM, permanent pacemaker.

Table 3 Multivariate analysis of risk factors for PPM implantation after HTX

\begin{tabular}{|c|c|c|c|}
\hline Variable & Hazard ratio & $95 \%$ confidence interval & $P$ value \\
\hline Recipient body mass index $\left(\mathrm{kg} / \mathrm{m}^{2}\right)$ & 1.10 & $1.01-1.21$ & $0.03^{*}$ \\
\hline Recipient arterial hypertension (in total) & 1.43 & $0.65-3.14$ & 0.37 \\
\hline Donor age (years) & 1.07 & $1.03-1.10$ & $<0.01^{*}$ \\
\hline
\end{tabular}

*, statistically significant $(\mathrm{P}<0.05)$. HTX, heart transplantation; PPM, permanent pacemaker.

after HTX included the following five parameters: recipient age in years (HR: 1.03; 95\% CI: 0.98-1.08; $\mathrm{P}=0.20$ ), recipient $\mathrm{BMI}$ in $\mathrm{kg} / \mathrm{m}^{2}$ (HR: 1.10; 95\% CI: 1.01-1.21; $\mathrm{P}=0.03$ ), recipient arterial hypertension (HR: $1.43 ; 95 \%$ CI: 0.65-3.14; $\mathrm{P}=0.37$ ), donor age in years (HR: 1.07 ; $95 \%$ CI: $1.03-1.10 ; \mathrm{P}<0.01$ ), and biatrial HTX (HR: 2.63; 95\% CI: 1.22-5.68; $\mathrm{P}=0.01)$. Multivariate analysis of risk factors for PPM implantation after HTX is provided in Table 3.

\section{Indications, perioperative data and complications of PPM implantation after HTX}

Indications for PPM implantation after HTX included 15 patients with SND (41.7\%) and 21 patients with
AVB (58.3\%). Patients with early PPM after HTX had a significantly higher rate of SND (66.7\% versus $29.2 \%$; $\mathrm{P}=0.03)$, whereas patients with late PPM after HTX showed a higher percentage of AVB (70.8\% versus $33.3 \%$; $\mathrm{P}=0.03)$ as indication for PPM after HTX.

Perioperative data and complications showed no significant differences between patients with early and late PPM after HTX. Indications, perioperative data and complications of PPM implantation after HTX are shown in Table 4.

\section{Follow-up measures of PPM implantation after HTX}

Patients with early PPM after HTX revealed a significantly 
Table 4 Indications, perioperative data and complications of PPM implantation after HTX

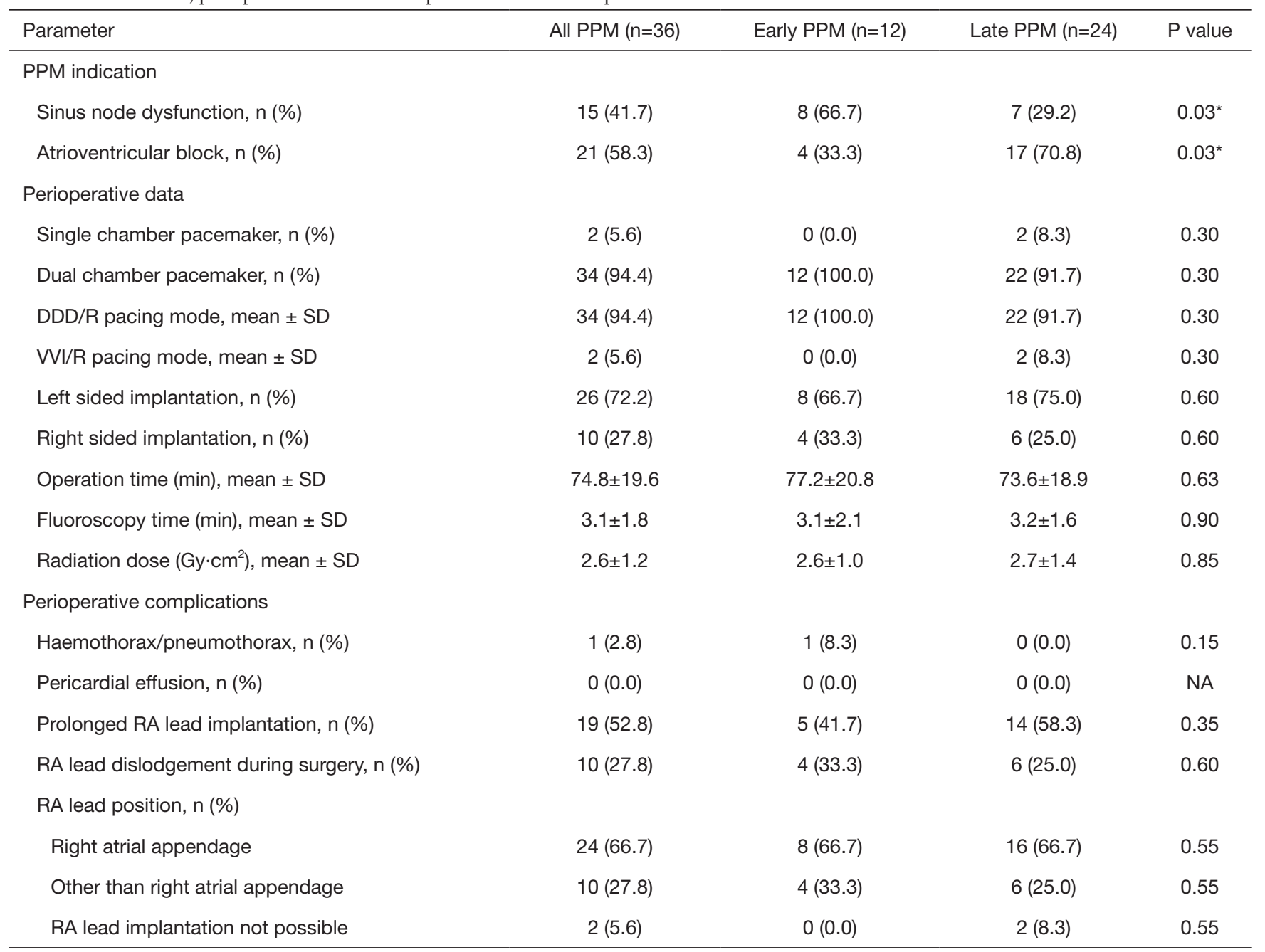

*, statistically significant $(\mathrm{P}<0.05)$. HTX, heart transplantation; $n$, number; NA, not applicable; PPM, permanent pacemaker; RA, right atrium; RV, right ventricle; SD, standard deviation.

lower percentage of atrial and ventricular pacing than patients with late PPM after HTX at baseline (atrial: $8.0 \% \pm 4.8 \%$ versus $20.9 \% \pm 26.9 \%$; $=0.04$; respectively ventricular: $19.4 \% \pm 21.5 \%$ versus $42.5 \% \pm 41.9 \%$; $\mathrm{P}=0.04$ ), at 6 -month follow-up (atrial: $2.0 \% \pm 1.7 \%$ versus $25.0 \% \pm 30.2 \% ; \mathrm{P}<0.01$; respectively ventricular: $0.5 \% \pm 0.8 \%$ versus $34.0 \% \pm 43.0 \% ; \mathrm{P}<0.01$ ), and at 24 -month followup (atrial: $1.3 \% \pm 1.1 \%$ versus $21.9 \% \pm 29.2 \% ; \mathrm{P}=0.03$; respectively ventricular: $0.3 \% \pm 0.3 \%$ versus $45.6 \% \pm 47.5 \%$; $\mathrm{P}<0.01)$.

No statistically significant differences could be observed between groups in regard to stimulation threshold, sensitivity, or impedance during follow-up. Follow-up measures of PPM implantation after HTX are provided in Table 5.

\section{Survival after $H T X$}

Patients with and without PPM implantation after HTX showed a similar 5 -year post-transplant survival in the Kaplan-Meier estimator indicating no effects of PPM implantation after HTX on post-transplant survival $(\mathrm{P}=0.85)$. Stratified by early $\mathrm{PPM}(\mathrm{PPM} \leq 1$ year after HTX), late PPM (PPM >1 year after HTX), and no PPM after HTX, the Kaplan-Meier estimator displayed a statistically significant inferior 5-year post-transplant survival of patients with early PPM after HTX in comparison to patients with late PPM or no PPM after 
Table 5 Follow-up measures of PPM implantation after HTX

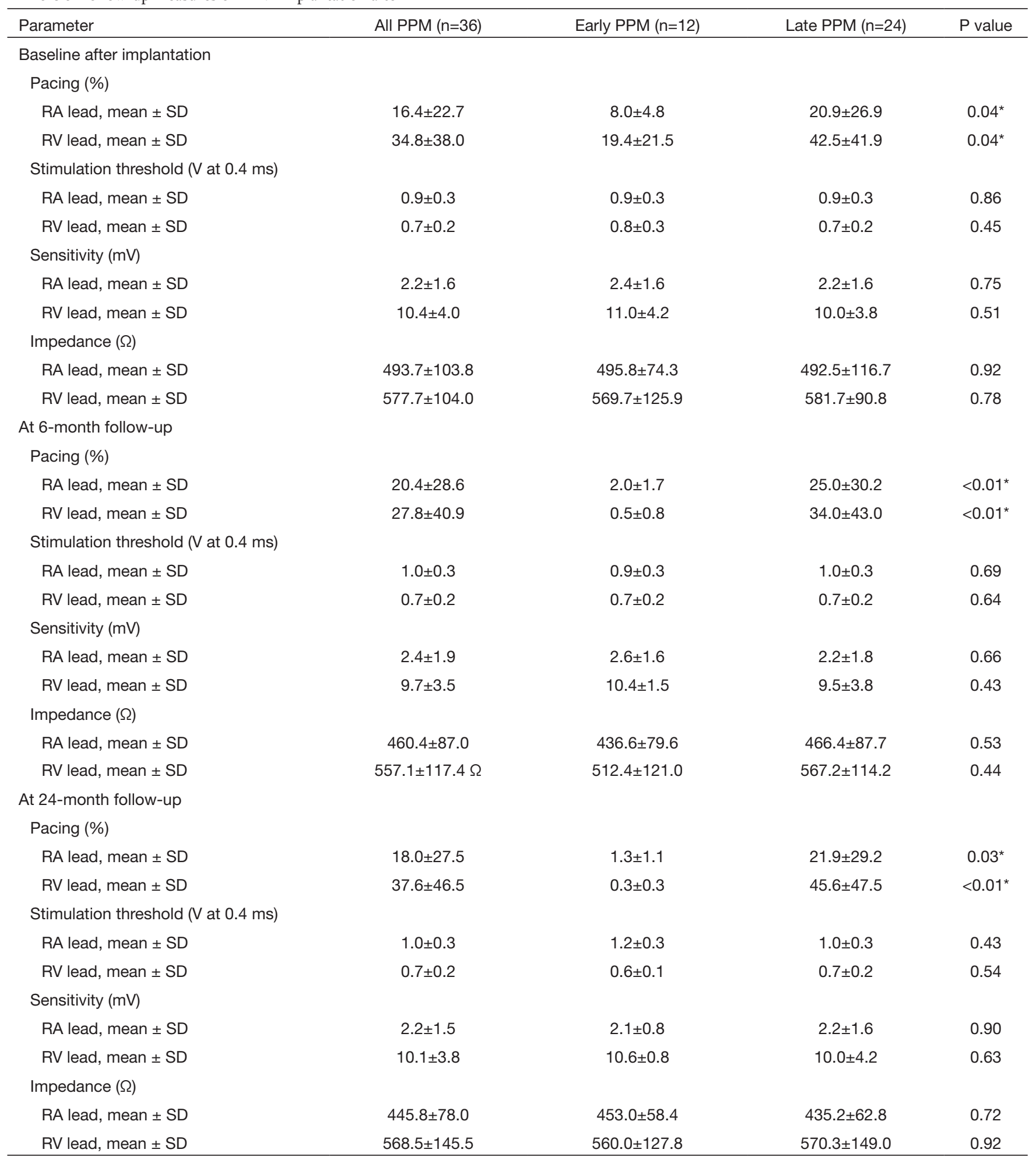

*, statistically significant $(\mathrm{P}<0.05)$. HTX, heart transplantation; PPM, permanent pacemaker; RA, right atrium; RV, right ventricle; SD, standard deviation. 


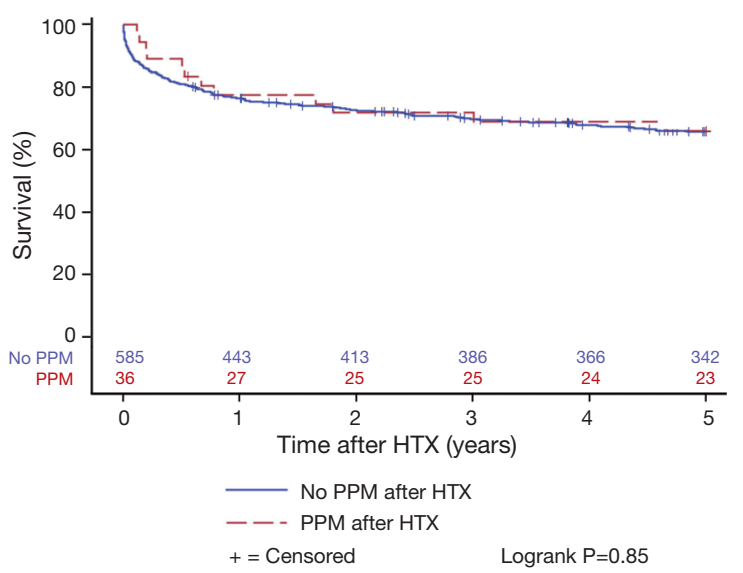

Figure 1 Post-transplant survival of patients with and without PPM after HTX (Kaplan-Meier estimator). There was no statistically significant difference in 5-year post-transplant survival between patients with and without PPM after HTX $(\mathrm{P}=0.85)$. HTX, heart transplantation; PPM, permanent pacemaker.

HTX $(\mathrm{P}<0.01)$. Kaplan-Meier estimators are presented in Figures 1 and 2.

\section{Causes of death after HTX}

A total of 12 patients with PPM implantation after HTX (33.3\%) deceased within five years after HTX. In the early PPM after HTX group, 9 patients (75.0\%) passed away, while 3 patients $(12.5 \%)$ deceased in the late PPM group after HTX. With regard to the causes of death, significantly more patients in the early PPM after HTX group died from infection/sepsis following chest infection than patients in the late PPM after HTX group (58.3\% versus $8.3 \%$; $\mathrm{P}<0.01$ ), while there was no significant difference in left ventricular ejection fraction. Moreover, there was no difference between groups in terms of transplant failure, acute rejection, malignancy, or thromboembolic event/ bleeding. Causes of death after HTX are given in Table 6.

\section{Sensitivity analysis}

A sensitivity analysis to test the robustness of the study results was performed with a subgroup of patients receiving TAC and MMF as immunosuppressive medication (277 of 621 patients, $44.6 \%)$. The robustness of the study results was confirmed as similar results were observed concerning risk factors and survival of patients with PPM after HTX.

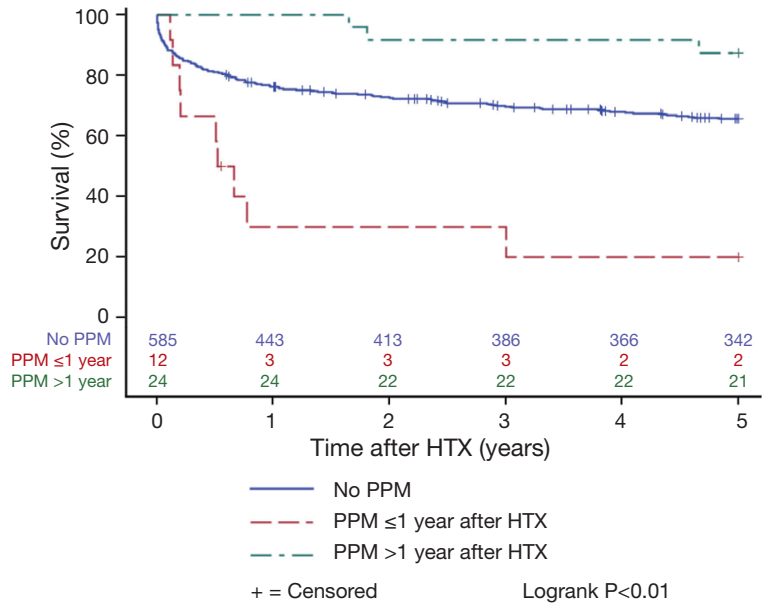

Figure 2 Post-transplant survival of patients with early PPM, late PPM, and no PPM after HTX (Kaplan-Meier estimator). Overview of 5-year post-transplant survival of patients stratified by early PPM (PPM $\leq 1$ year after HTX), late PPM (PPM >1 year after HTX), and no PPM after HTX. Patients with early PPM after HTX showed a statistically significant inferior 5-year posttransplant survival in comparison to patients with late $\mathrm{PPM}$ or no PPM after HTX $(\mathrm{P}<0.01)$. HTX, heart transplantation; PPM, permanent pacemaker.

\section{Discussion}

\section{Indications for and timing of PPM implantation after HTX}

More than $50 \%$ of patients after HTX experience bradyarrhythmias within the first few post-transplant weeks $(6,20,36)$. SND is the most common cause of bradyarrhythmia in the initial post-transplant period while AVB tends to present later after $\operatorname{HTX}(1,6,8,13,37)$.

In the event of bradyarrhythmias after HTX, reversible causes such as negative chronotropic drugs, electrolyte imbalance, or hypothyreosis should be addressed first, followed by heart rate stimulating agents and temporary pacing considering PPM implantation as last option $(6,12,28,36)$. If inevitable, the optimal timing of PPM implantation is essential in managing the balancing act between the early need for pacing and the risk of potential complications $(6,12)$. The extended use of epicardial temporary pacing may be an adequate solution to bridge patients over transient bradyarrhythmias during the initial period after $\operatorname{HTX}(6,12,36)$. This could-in combination with other measurements such as the use of 
Table 6 Causes of death within 5 years after HTX

\begin{tabular}{|c|c|c|c|c|}
\hline Parameter & All PPM $(n=36)$ & Early PPM (n=12) & Late PPM $(n=24)$ & $P$ value \\
\hline Acute rejection, n (\%) & $0(0.0)$ & $0(0.0)$ & $0(0.0)$ & NA \\
\hline Infection/sepsis, n (\%) & $9(25.0)$ & 7 (58.3) & $2(8.3)$ & $<0.01^{*}$ \\
\hline Malignancy, n (\%) & $0(0.0)$ & $0(0.0)$ & $0(0.0)$ & NA \\
\hline All causes, n (\%) & $12(33.3)$ & $9(75.0)$ & $3(12.5)$ & $<0.01^{*}$ \\
\hline
\end{tabular}

*, statistically significant $(\mathrm{P}<0.05)$. HTX, heart transplantation; $n$, number; NA, not applicable; PPM, permanent pacemaker.

bicaval HTX instead of biatrial HTX-effectively reduce the incidence of PPM implantation due to SND in the early period after HTX. Additionally, regarding long term follow-up, the focus of PPM implantation after HTX could consequently change from SND early after HTX towards AVB in a later post-transplant setting. However, temporary pacing comes at a price such as an increased risk for infection, lead dislodgement, inadequate pacing, and may not always be possible in patients with rejection or voluminous fibrosis (6).

In this study, the need for overall PPM implantation after HTX $(5.8 \%)$ was comparatively lower in patients with an extended use of epicardial temporary pacing combined with a predominant use of bicaval HTX. Additionally, the rate of early PPM implantation after HTX could be reduced to $1.9 \%$ (33.3\% of all PPM implantations after HTX).

\section{Risk factors for PPM implantation after HTX}

As the causes for PPM implantation after HTX are still not fully understood, this large registry study with 621 patients investigated potential risk factors in detail. Our multivariate analysis showed an elevated recipient BMI, a higher donor age, and the performance of biatrial HTX as significant risk factors for PPM implantation after HTX. This is in line with findings by Cantillon et al. (5) who identified biatrial surgical technique and increasing donor age as important associations with the occurrence of bradyarrhythmias and requirement for PPM implantation after HTX in a large multi-center study.

To our knowledge, this is the first study to show an elevated recipient BMI as an independent risk factor for PPM implantation after HTX. The occurrence of bradyarrhythmias has been linked to obesity and sleep apnea $(52,53)$. Cessation of breathing and hypoxemia are postulated to be essential factors in the emergence of bradyarrhythmias (53). Additionally, due to cardiac denervation in patients after HTX, the autonomous control of the heart is affected making these patients more vulnerable to changes in chronotropic function (45).

During the study period from 1989 to 2018, we found no relevant imbalance in PPM implantations after HTX reducing the likelihood of a potential era effect.

Prolonged ischemic time has previously been reported to be associated with bradyarrhythmias after HTX as hypoxia during surgery can cause damage to the sinus node and the electrical conduction system of the cardiac allograft $(1,4,28)$. However, we and other recent studies could not detect a significant association between ischemic time and PPM implantation after HTX (5,7,11-13).

Pre-transplant use of amiodarone has been suggested to be another potential risk factor for PPM implantation after HTX (6). In a recent study, our group could show that neither short-term nor long-term amiodarone use before HTX was related to post-transplant bradycardia or PPM implantation after HTX (47). These results were supported by findings by Zieroth et al. (7) and by Woo et al. (43) who found no statistically significant association between pretransplant amiodarone use and the requirement for PPM implantation after HTX. In addition, negative chronotropic drugs may cause a relevant heart rate reduction. However, we could not detect statistically significant differences between patients with and without PPM implantation after HTX regarding the administration of beta blockers, calcium channel blockers or ivabradine in this study.

\section{Post-transplant survival and causes of death}

Due to the lack of organ donation, it is essential to continuously improve quality of life and to search for 
risk factors which may impair survival after HTX (11). Patients after HTX require an immunosuppressive drug regimen to prevent acute rejection episodes $(50,51)$. These patients are consequently more vulnerable to infections especially in the initial post-transplant period when they require higher levels of immunosuppressive drugs $(44,50,51)$. Hence, it is clinically very important to know whether PPM implantation after HTX is associated with increased post-transplant mortality. We observed a similar 5-year post-transplant survival between patients with and without PPM implantation after HTX in general. In accordance with our findings, several other authors found no significant association between overall PPM implantation after HTX and post-transplant mortality $(3,4,13,15,27,28)$.

Although overall PPM implantation does not seem to be related to increased post-transplant mortality, further stratification into patients with early and late PPM after HTX may reveal relevant differences which may have an impact on post-transplant survival. Unfortunately, as a result of sample sizes, only a couple of studies adopted this approach $(4,8)$. Cantillon et al. (4) detected no significant difference in 5 -year post-transplant survival between patients with early (61 patients), late (45 patients) and no (1,201 patients) PPM implantation after HTX. Likewise, Jones et al. (8) reported no significant difference in 5-year post-transplant survival between patients with early (30 patients), late (18 patients) and no (341 patients) PPM implantation after HTX. In contrast, we found a statistically significant inferior 5-year post-transplant survival of patients with early (12 patients) PPM after HTX in comparison to patients with late (24 patients) or no (585) PPM after HTX $(\mathrm{P}<0.01)$ along with a higher percentage of death due to infection $(\mathrm{P}<0.01)$.

When comparing these three large studies, differences in the overall rate of PPM implantation after HTX (Cantillon: $8.1 \%$; Jones: $12.3 \%$, this study: $5.8 \%$ ), the ratio of early versus (vs.) late PPM implantation after HTX (Cantillon: $57.5 \%$ vs. $42.5 \%$; Jones: $62.5 \%$ vs. $37.5 \%$, this study: $33.3 \%$ vs. $66.7 \%$ ), the definition of early PPM implantation after HTX (Cantillon: $\leq 30$ days after HTX; Jones: $\leq 30$ days after HTX, this study: $\leq 1$ year after HTX), and the location of study (Cantillon: USA; Jones: UK, this study: Germany) should be carefully considered $(4,8)$. Therefore, given the few numbers of studies analyzing differences in mortality between early and late PPM implantation after HTX, further large multi-center trials are necessary.

\section{Study limitations}

Our findings were derived from a single-center registry study (Heidelberg HTX Registry) with 621 adult patients receiving HTX at Heidelberg Heart Center. Based on the study design, results should be treated with caution as it carries certain limitations. However, our study provides an excellent granularity which most multi-center studies lack. Further, patients received a standardized center-specific pre-, peri-, and post-transplant course of treatment and follow-up reducing the likelihood of potential selection bias and confounders (2,44-51).

This study analyzed data from patients receiving HTX at the Heidelberg Heart Center between 1989 and 2018. Due to this long study period, a possible era effect as a result of changes in medical care cannot be ruled out. In order to test the robustness of the study results, a sensitivity analysis including patients with TAC and MMF was carried out as the immunosuppressive drug therapy was subsequently switched from CsA and MMF to TAC and MMF from 2006 onward. Here, similar results were observed. A further change of medical treatment within the study period was the use of ivabradine instead of beta blockers or calcium channel blockers for heart rate reduction in patients after HTX from 2006 onward. However, there were no significant differences between patients with and without PPM implantation after HTX regarding the use of ivabradine, beta blockers or calcium channel blockers (45).

Our results should be regarded as hypothesis-generating, especially with respect to survival as multiple factors may influence survival. Hence, our data cannot proof or disproof a causal relationship between early PPM implantation after HTX and impaired post-transplant survival but merely indicate an association, especially in view of the relatively small number of patients with PPM implantation after HTX (2,44-51). Hence, to confirm our results, further large multi-center trials are desirable to investigate risk factors and survival of patients with PPM implantation after HTX.

\section{Conclusions}

Our data showed a comparatively lower need for PPM implantation after HTX in patients with an extended use of epicardial temporary pacing for up to 10 days combined with a predominant use of bicaval HTX. Overall PPM implantation after HTX was necessary in only $5.8 \%$ of patients. Additionally, the rate of early PPM implantation after HTX ( $\leq 1$ year after HTX) could be reduced to $1.9 \%$ 
(33.3\% of all PPM implantations after HTX).

Multivariate analysis indicated a higher recipient BMI, an increased donor age, and the use of biatrial HTX as significant risk factors for PPM implantation after HTX. Kaplan-Meier estimator showed a significant inferior 5-year post-transplant survival of patients with early PPM after HTX in comparison to patients with late PPM or no PPM after HTX along with a higher percentage of death due to infection.

In summary, multivariate risk factors for PPM implantation after HTX include recipient BMI, donor age, and biatrial HTX. Early PPM implantation after HTX is associated with increased 5-year post-transplant mortality due to infection.

\section{Acknowledgments}

We acknowledge financial support by Deutsche Forschungsgemeinschaft within the funding program Open Access Publishing, by the Baden-Württemberg Ministry of Science, Research and the Arts and by the University of Heidelberg. Rasmus Rivinius is funded by research grants from the Faculty of Medicine, University of Heidelberg (Physician-Scientist-Program Scholarship) and the German Cardiac Society (Research Scholarship). Ann-Kathrin Rahm is supported by the German Society of Internal Medicine (Clinician-Scientist-Program Scholarship). We thank Anna Daut, Viola Deneke and Berthold Klein for their assistance and advice.

\section{Footnote}

Conflicts of Interest: The authors have no conflicts of interest to declare.

Ethical Statement: The authors are accountable for all aspects of the work in ensuring that questions related to the accuracy or integrity of any part of the work are appropriately investigated and resolved. This work complies with the Declaration of Helsinki. The study was approved by the ethics committee of the University of Heidelberg (S286/2015). Written informed consent was obtained from patients for inclusion in the Heidelberg HTX Registry allowing the clinical and scientific use of data.

\section{References}

1. Jacquet L, Ziady G, Stein K, et al. Cardiac rhythm disturbances early after orthotopic heart transplantation: prevalence and clinical importance of the observed abnormalities. J Am Coll Cardiol 1990;16:832-7.

2. Rivinius R, Helmschrott M, Ruhparwar A, et al. The influence of surgical technique on early posttransplant atrial fibrillation-comparison of biatrial, bicaval, and total orthotopic heart transplantation. Ther Clin Risk Manag 2017;13:287-97.

3. DiBiase A, Tse TM, Schnittger I, et al. Frequency and mechanism of bradycardia in cardiac transplant recipients and need for pacemakers. Am J Cardiol 1991;67:1385-9.

4. Cantillon DJ, Gorodeski EZ, Caccamo M, et al. Longterm outcomes and clinical predictors for pacing after cardiac transplantation. J Heart Lung Transplant 2009;28:791-8.

5. Cantillon DJ, Tarakji KG, Hu T, et al. Long-term outcomes and clinical predictors for pacemaker-requiring bradyarrhythmias after cardiac transplantation: analysis of the UNOS/OPTN cardiac transplant database. Heart Rhythm 2010;7:1567-71.

6. Bacal F, Bocchi EA, Vieira ML, et al. Permanent and temporary pacemaker implantation after orthotopic heart transplantation. Arq Bras Cardiol 2000;74:9-12.

7. Zieroth S, Ross H, Rao V, et al. Permanent pacing after cardiac transplantation in the era of extended donors. J Heart Lung Transplant 2006;25:1142-7.

8. Jones DG, Mortsell DH, Rajaruthnam D, et al. Permanent pacemaker implantation early and late after heart transplantation: clinical indication, risk factors and prognostic implications. J Heart Lung Transplant 2011;30:1257-65.

9. Kara I, Ay Y, Yanarta M, et al. Does the surgical technique used in the orthotopic heart transplant affect the results regarding the rhythm? Anadolu Kardiyol Derg 2012;12:255-60.

10. Thompson MA, Patel H. Posttransplant pacemaker placement: case series and review. Ochsner J 2010;10:236-40.

11. Herrmann FEM, Wellmann P, Sadoni S, et al. Sinus node dysfunction after heart transplantation-An analysis of risk factors and atrial pacing burden. Clin Transplant 2018;32:e13202.

12. Mallidi HR, Bates M. Pacemaker Use Following Heart Transplantation. Ochsner J 2017;17:20-4.

13. Wellmann P, Herrmann FE, Hagl C, et al. A Single Center Study of 1,179 Heart Transplant Patients-Factors Affecting Pacemaker Implantation. Pacing Clin Electrophysiol 2017;40:247-54. 
14. Noworolski R, Przybylowski P, Majewski J, et al. Early and late indications for implantation of cardiac pacemakers in patients after heart transplantation: a single-center experience. Transplant Proc 2011;43:3074-5.

15. Tay AE, Faddy S, Lim S, et al. Permanent pacing for late-onset atrioventricular block in patients with heart transplantation: a single center experience. Pacing Clin Electrophysiol 2011;34:72-5.

16. Blanche C, Czer LS, Fishbein MC, et al. Permanent pacemaker for rejection episodes after heart transplantation: a poor prognostic sign. Ann Thorac Surg 1995;60:1263-6.

17. Buja G, Miorelli M. Livi U, et al. Complete atrioventricular block after orthotopic heart transplantation: Incidence, electrocardiographic-electrophysiological evolution and clinical importance. Eur J Card Pacing Electrophysiol 1992;3:173-9.

18. Cataldo R, Olsen S, Freedman RA. Atrioventricular block occurring late after heart transplantation: presentation of three cases and literature review. Pacing Clin Electrophysiol 1996;19:325-30.

19. Chau EM, McGregor CG, Rodeheffer RJ, et al. Increased incidence of chronotropic incompetence in older donor hearts. J Heart Lung Transplant 1995;14:743-8.

20. Cooper MM, Smith CR, Rose EA, et al. Permanent pacing following cardiac transplantation. J Thorac Cardiovasc Surg 1992;104:812-6.

21. Grant SC, Khan MA, Faragher EB, et al. Atrial arrhythmias and pacing after orthotopic heart transplantation: bicaval versus standard atrial anastomosis. Br Heart J 1995;74:149-53.

22. Heinz G, Hirschl M, Buxbaum P, et al. Sinus node dysfunction after orthotopic cardiac transplantation: postoperative incidence and long-term implications. Pacing Clin Electrophysiol 1992;15:731-7.

23. Heinz G, Kratochwill C, Koller-Strametz J, et al. Benign prognosis of early sinus node dysfunction after orthotopic cardiac transplantation. Pacing Clin Electrophysiol 1998;21:422-9.

24. Holt ND, McComb JM. Late atrioventricular (AV) block and permanent pacemaker implantation after orthotopic cardiac transplantation. Pacing Clin Electrophysiol 1996;19:1272.

25. Kratochwill C, Schmid S, Koller-Strametz J, et al. Decrease in pacemaker incidence after orthotopic heart transplantation. Am J Cardiol 1996;77:779-83.

26. Luciani GB, Faggian G, Montalbano G, et al. Orthotopic heart transplantation with bicaval anastomosis using older donors. Transplant Proc 1997;29:3389-91.

27. Markewitz A, Schmoeckel M, Nollert G, et al. Longterm results of pacemaker therapy after orthotopic heart transplantation. J Card Surg 1993;8:411-6.

28. Miyamoto Y, Curtiss EI, Kormos RL, et al. Bradyarrhythmia after heart transplantation. Incidence, time course, and outcome. Circulation 1990;82:IV313-7.

29. Montero JA, Anguita M, Concha M, et al. Pacing requirements after orthotopic heart transplantation: incidence and related factors. J Heart Lung Transplant 1992;11:799-802.

30. Parry G, Holt ND, Dark JH, et al. Declining need for pacemaker implantation after cardiac transplantation. Pacing Clin Electrophysiol 1998;21:2350-2.

31. Payne ME, Murray KD, Watson KM, et al. Permanent pacing in heart transplant recipients: underlying causes and long-term results. J Heart Lung Transplant 1991;10:738-42.

32. Raghavan C, Maloney JD, Nitta J, et al. Long-term followup of heart transplant recipients requiring permanent pacemakers. J Heart Lung Transplant 1995;14:1081-9.

33. Redmond JM, Zehr KJ, Gillinov MA, et al. Use of theophylline for treatment of prolonged sinus node dysfunction in human orthotopic heart transplantation. J Heart Lung Transplant 1993;12:133-8.

34. Roelke M, McNamara D, Osswald S, et al. A comparison of VVIR and DDDR pacing following cardiac transplantation. Pacing Clin Electrophysiol 1994;17:2047-51.

35. Rothman SA, Jeevanandam V, Combs WG, et al. Eliminating bradyarrhythmias after orthotopic heart transplantation. Circulation 1996;94:II278-82.

36. Scott CD, McComb JM, Dark JH, et al. Permanent pacing after cardiac transplantation. Br Heart J 1993;69:399-403.

37. Scott CD, Omar I, McComb JM, et al. Long-term pacing in heart transplant recipients is usually unnecessary. Pacing Clin Electrophysiol 1991;14:1792-6.

38. Weinfeld MS, Kartashov A, Piana R, et al. Bradycardia: a late complication following cardiac transplantation. Am J Cardiol 1996;78:969-71.

39. Woodard DA, Conti JB, Mills RM Jr, et al. Permanent atrial pacing in cardiac transplant patients. Pacing Clin Electrophysiol 1997;20:2398-404.

40. Herre JM, Barnhart GR, Llano A. Cardiac pacemakers in the transplanted heart: short term with the biatrial anastomosis and unnecessary with the bicaval anastomosis. Curr Opin Cardiol 2000;15:115-20.

41. Luebbert JJ, Lee FA, Rosenfeld LE. Pacemaker therapy for 
early and late sinus node dysfunction in orthotopic heart transplant recipients: a single-center experience. Pacing Clin Electrophysiol 2008;31:1108-12.

42. Meyer SR, Modry DL, Bainey K, et al. Declining need for permanent pacemaker insertion with the bicaval technique of orthotopic heart transplantation. Can J Cardiol 2005;21:159-63.

43. Woo GW, Schofield RS, Pauly DF, et al. Incidence, predictors, and outcomes of cardiac pacing after cardiac transplantation: an 11-year retrospective analysis. Transplantation 2008;85:1216-8.

44. Rivinius R, Helmschrott M, Ruhparwar A, et al. COPD in patients after heart transplantation is associated with a prolonged hospital stay, early posttransplant atrial fibrillation, and impaired posttransplant survival. Clin Epidemiol 2018;10:1359-69.

45. Rivinius R, Helmschrott M, Ruhparwar A, et al. Control of cardiac chronotropic function in patients after heart transplantation: effects of ivabradine and metoprolol succinate on resting heart rate in the denervated heart. Clin Res Cardiol 2018;107:138-47.

46. Rivinius R, Helmschrott M, Ruhparwar A, et al. Chronic digitalis therapy in patients before heart transplantation is an independent risk factor for increased post-transplant mortality. Ther Clin Risk Manag 2017;13:1399-407.

47. Rivinius R, Helmschrott M, Ruhparwar A, et al. Comparison of posttransplant outcomes in patients

Cite this article as: Rivinius $\mathrm{R}$, Helmschrott $M$, Rahm AK, Darche FF, Thomas D, Bruckner T, Doesch AO, Ehlermann P, Katus HA, Zitron E. Risk factors and survival of patients with permanent pacemaker implantation after heart transplantation. J Thorac Dis 2019;11(12):5440-5452. doi: 10.21037/jtd.2019.11.45 with no, acute, or chronic amiodarone use before heart transplantation. Drug Des Devel Ther 2017;11:1827-37.

48. Rivinius R, Helmschrott M, Ruhparwar A, et al. Longterm use of amiodarone before heart transplantation reduces significantly early post-transplant atrial fibrillation and is not associated with increased mortality after heart transplantation. Drug Des Devel Ther 2016;10:677-86.

49. Rivinius R, Helmschrott M, Ruhparwar A, et al. Analysis of malignancies in patients after heart transplantation with subsequent immunosuppressive therapy. Drug Des Devel Ther 2014;9:93-102.

50. Helmschrott M, Rivinius R, Ruhparwar A, et al. Advantageous effects of immunosuppression with tacrolimus in comparison with cyclosporine A regarding renal function in patients after heart transplantation. Drug Des Devel Ther 2015;9:1217-24.

51. Helmschrott M, Rivinius R, Bruckner T, Katus HA, Doesch AO. Renal function in heart transplant patients after switch to combined mammalian target of rapamycin inhibitor and calcineurin inhibitor therapy. Drug Des Devel Ther 2017;11:1673-80.

52. Frank S, Colliver JA, Frank A. The electrocardiogram in obesity: statistical analysis of 1,029 patients. J Am Coll Cardiol 1986;7:295-9.

53. Daccarett M, Segerson NM, Hamdan AL, et al. Relation of daytime bradyarrhythmias with high risk features of sleep apnea. Am J Cardiol 2008;101:1147-50. 